\title{
Faktor Koreksi Diagram Kendali Shewhart pada Situasi Unconditional ARL dan Penerapannya terhadap Data Brix (Kekentalan) Saus
}

\author{
Riani Shifa Rahmadani*, Suliadi \\ Prodi Statistika, Fakultas Matematika dan Ilmu Pengetahuan Alam, \\ Universitas Islam Bandung, Indonesia.
}

*syiparahmadani@gmail.com, suliadi@unisba.ac.id

\begin{abstract}
Statistical Proses Control (SPC) is application of method in statistics on measurements and analysis variation in proses. Shewhart control chart is ussually used as a device in SPC. In practice, the parameters prosses are usually unknown. The parameters prosses is estimated using data often from the previous processes, ARL of the control chart with estimated parameters as called as unconditional ARL. Goedhart et al propose a new correction factor to improve unconditional ARL such data unconditional ARL close to the expected ARL. In this articel we apply the correction factor of Goedhart to brix's data of PT. XXX. We offten the obtained the upper control limit is 39.51 , the lower control limit is 36.87 with mean 38.19 applying this control limit to brix's data results that the proses is under control.
\end{abstract}

Keywords: ARL, Goedhart correction factors, Brix Sauce.

Abstrak. Statistical Proses Control (SPC) merupakan penerapan metode statistik dalam pengukuran dan analisis variasi proses. Alat yang sering digunakan dalam metode SPC yaitu diagram kendali Shewhart. Dalam praktiknya, parameter proses biasanya tidak diketahui. Parameter proses tersebut biasanya diestimasi melalui data yang diperoleh dari proses sebelumnya, ARL untuk diagram kendali dengan parameter yang diestimasi dinamakan ARL unconditional. Goedhart dkk mengusulkan faktor koreksi baru untuk meningkatkan ARL unconditional yang memiliki ekspetasi sebesar ARL yang diharapkan. Dalam artikel ini kami menerapkan faktor koreksi Goedhart terhadap data brix PT. XXX dan diperoleh batas kendali atas 39.51, batas kendali bawah 36.87 dengan rata-rata 38.19 Dari implementasi diagram kendali terhadap data kekentalan diperoleh bahwa proses terkendali.

Kata Kunci: ARL, Faktor Koreksi Goedhart, Brix Saus. 


\section{A. Pendahuluan}

Salah satu alat yang dapat digunakan dalam mengontrol proses yaitu SPC dengan alat utamanya yaitu diagram kendali Shewhart. Menurut Montgomery (1990) diagram kendali Shewhart dapat digunakan untuk memonitor proses dengan mengidentifikasi variasi-variasi yang ada didalam proses apakah variasi normal atau tidak normal. Dalam praktiknya, parameter proses dalam diagram kendali Shewhart biasanya diperoleh dari estimasi. Dalam diagram kendali Shewhart nilai ARL yang didapatkan dari estimasi parameter dinamakan ARL unconditional. Untuk mengatasi masalah tersebut maka diperlukan faktor koreksi. Sebelumnya Albers dan Kallenberg (2005) membuat faktor koreksi dimana nilai ARL unconditional jauh dari ARL yang diharapkan maka dari itu Goedhart mengusulkan faktor koreksi yang lebih baik dimana nilai ARL unconditional dekat dengan nilai ARL yang diharapkan.

Pada artikel ini kami menggunakannya pada faktor koreksi Goedhart dan menerapkannya pada data brix saus (kekentalan saus) PT. XXX untuk menyusun diagram kendali Shewhart. Dari penjelasan diatas didapatkan bahwa masalah yang dihadapi yaitu : "Bagaimana cara estimasi parameter untuk diagram kendali Shewhart terhadap data brix saus (kekentalan saus) ?" dan juga "Bagaimana membuat faktor koreksi baru untuk diagram kendali Shewhart pada kondisi ARL unconditional terhadap data brix saus (kekentalan saus)?" dengan tujuan sebagai berikut:

1. Untuk estimasi parameter diagram kendali Shewhart terhadap data brix saus (kekentalan saus).

2. Untuk membuat faktor koreksi diagram kendali Shewhart pada kondisi ARL unconditional terhadap data brix saus (kekentalan saus).

\section{B. Landasan Teori}

Menurut Goedhart dkk (2016) ARL dapat memantau kinerja dari diagram kendali Shewhart. Ada dua jenis ARL yaitu ARL berdasarkan parameter diketahui yang disebut ARL Conditional (CARL) dan ARL unconditional (EARL) jika parameter yang tidak diketahui.

Misalkan $E_{i}$ merupakan kejadian $\bar{Y}_{l}$ yang keluar dari batas kendali sehingga,

$$
P\left(E_{i} \mid \hat{\mu}, \hat{\sigma}\right)=P\left(\bar{Y}_{l}<\widehat{L C L}\right)+P\left(\bar{Y}_{l}>\widehat{U C L}\right)
$$

Dengan nilai $\mu$ dan $\sigma$ digantikan oleh estimasi tak bias $\hat{\mu}$ dan $\hat{\sigma}$ untuk nilai $\widehat{L C L}$ dan $\widehat{U C L}$.Goedhart dkk (2016) mendefinisikan nilai $\mathrm{CARL}=\frac{1}{P\left(E_{i} \mid \hat{\mu}, \hat{\sigma}\right)}$ dan nilai $\mathrm{EARL}=$ $E\left(\frac{1}{P\left(E_{i} \mid \hat{\mu}, \hat{\sigma}\right)}\right)=E(C A R L)$ dan mengusulkan batas kendali baru yaitu,

$$
\widehat{U C L}=\hat{\mu}+\frac{\widetilde{K} \widehat{\sigma}}{\sqrt{n}} \text { dan } \widehat{L C L}=\hat{\mu}-\frac{\widetilde{K} \widehat{\sigma}}{\sqrt{n}}
$$

Dimana $\widetilde{K}=K+c$ dengan $c$ adalah faktor koreksi baru untuk mencapai EARL yang diinginkan pada kondisi proses terkendali. Untuk mendapatkan nilai yang akurat dalam mengestimasi nilai $c$. Maka nilai $c$ diperoleh melalui probabilitas bersyarat dimana $P\left(E_{i} \mid \hat{\mu}, \hat{\sigma}\right)$ diberikan oleh,

$$
\begin{aligned}
P\left(E_{i} \mid \hat{\mu}, \hat{\sigma}\right) & =1-P\left(\widehat{L C L}<\bar{Y}_{\iota}<\widehat{U C L}\right) \\
P\left(E_{i} \mid \hat{\mu}, \hat{\sigma}\right) & =1-\left[P\left(\frac{\hat{\mu}-\widetilde{K} \hat{\sigma}}{\sqrt{n}}<\bar{Y}_{\iota}<\frac{\hat{\mu}+\widetilde{K} \hat{\sigma}}{\sqrt{n}}\right)\right] \\
& =1-\left[P\left(\bar{Y}_{l}<\frac{\hat{\mu}+\widetilde{K} \hat{\sigma}}{\sqrt{n}}\right)-P\left(\bar{Y}_{\iota}<\frac{\hat{\mu}-\widetilde{K} \hat{\sigma}}{\sqrt{n}}\right)\right]
\end{aligned}
$$

Karena $\bar{Y}_{\imath} \sim N\left(\mu, \frac{\sigma}{\sqrt{n}}\right)$ maka, 


$$
\begin{aligned}
P\left(E_{i} \mid \hat{\mu}, \hat{\sigma}\right) & =1-\left[P\left(\frac{\overline{Y_{l}}-\mu}{\sigma / \sqrt{n}}<\frac{\hat{\mu}+\widetilde{K} \hat{\sigma} / \sqrt{n}-\mu}{\sigma / \sqrt{n}}\right)-P\left(\frac{\overline{Y_{l}}-\mu}{\sigma / \sqrt{n}}<\frac{\hat{\mu}-\widetilde{K} \hat{\sigma} / \sqrt{n}-\mu}{\sigma / \sqrt{n}}\right)\right] \\
& =1-\left[\left(\Phi<\frac{\hat{\mu}-\mu}{\sigma / \sqrt{n}}+\widetilde{K} \frac{\hat{\sigma}}{\sigma}\right)-\left(\Phi<\frac{\hat{\mu}-\mu}{\sigma / \sqrt{n}}-\widetilde{K} \frac{\hat{\sigma}}{\sigma}\right)\right]
\end{aligned}
$$

Dengan $\widetilde{K}=K+c, \bar{\Phi}=1-\Phi(x), \Phi(x)$ adalah fungsi distribusi normal $(0,1)$. Misalkan,

dan

$$
\Delta_{1}(\widetilde{K})=\frac{\hat{\mu}-\mu}{\sigma / \sqrt{n}}+\widetilde{K} \frac{\hat{\sigma}}{\sigma}-\widetilde{K}
$$

Maka,

$$
\Delta_{2}(\widetilde{K})=-\frac{\hat{\mu}-\mu}{\sigma / \sqrt{n}}+\widetilde{K} \frac{\hat{\sigma}}{\sigma}-\widetilde{K}
$$

$$
\begin{aligned}
P\left(E_{i} \mid \hat{\mu}, \hat{\sigma}\right)= & {\left[1-\Phi\left(\widetilde{K}+\Delta_{1}(\widetilde{K})\right)\right]+\left[1-\Phi\left(\widetilde{K}+\Delta_{2}(\widetilde{K})\right)\right] } \\
=\bar{\Phi}\left(\widetilde{K}+\Delta_{1}(\widetilde{K})\right) & \\
& +\Phi(\widetilde{K} \\
& \left.+\Delta_{2}(\widetilde{K})\right)
\end{aligned}
$$

Notasi ini, menurut Goedhart dkk (2016) sama dengan Albers dan Kallenberg (2005) untuk faktor koreksi individu dan dapat dirubah menjadi fungsi $g$ untuk $P\left(E_{i} \mid \hat{\mu}, \hat{\sigma}\right)$,

$$
g\left(P\left(E_{i} \mid \hat{\mu}, \hat{\sigma}\right)\right)=h\left(\widetilde{K}+\Delta_{1}(\widetilde{K}), \widetilde{K}+\Delta_{2}(\widetilde{K})\right)=h_{(x, y)}
$$

Nilai dari $h_{(x, y)}$ merupakan notasi umum dalam perhitungan faktor koreksi.

Menurut Goedhart dkk (2016) nilai $\widetilde{K}$ tertentu dalam (2.3) bersifat konstant. Maka, $h\left(\widetilde{K}+\Delta_{1}(\widetilde{K}), \widetilde{K}+\Delta_{2}(\widetilde{K})\right)=h\left(x_{0}+\Delta x, y_{0}+\Delta y\right)$.Goedhart dkk (2016) juga mengestimasi $P\left(E_{i} \mid \hat{\mu}, \hat{\sigma}\right)$ menggunakan perluasan dua langkah dari Taylor,

$h\left(\widetilde{K}+\Delta_{1}(\widetilde{K}), \widetilde{K}+\Delta_{2}(\widetilde{K})\right)$

$\approx h(\widetilde{K}, \widetilde{K})+h_{x}(\widetilde{K}, \widetilde{K}) \Delta_{1}(\widetilde{K})+h_{y}(\widetilde{K}, \widetilde{K}) \Delta_{2}(\widetilde{K})$

$+\frac{1}{2}\left[h_{x x}(\widetilde{K}, \widetilde{K}) \Delta_{1}^{2}(\widetilde{K}, \widetilde{K})+2 h_{x y}(\widetilde{K}, \widetilde{K}) \Delta_{1}(\widetilde{K}), \Delta_{2}(\widetilde{K})+h_{y y}(\widetilde{K}, \widetilde{K}) \Delta_{2}^{2}(\widetilde{K}, \widetilde{K})\right]$

Menurut Goedhart dkk (2016), jika parameter diperoleh melalui estimasi maka nilai $E g\left(P\left(E_{i} \mid \hat{\mu}, \hat{\sigma}\right)\right) \neq g(\alpha)$. Untuk memastikan nilai untuk $E g\left(P\left(E_{i} \mid \hat{\mu}, \hat{\sigma}\right)\right)=g(\alpha)$ maka, harus dicari nilai $c$ dengan $\widetilde{K}=K+c$, melalui persamaan berikut ini,

$$
\begin{aligned}
E g\left(P\left(E_{i} \mid \hat{\mu}, \hat{\sigma}\right)\right)= & E h\left(\widetilde{K}+\Delta_{1}(\widetilde{K}), \widetilde{K}+\Delta_{2}(\widetilde{K})\right) \\
\approx h(\widetilde{K}, \widetilde{K})+h_{x}(\widetilde{K}, \widetilde{K})\left[E \Delta_{1}(\widetilde{K})+E \Delta_{2}(\widetilde{K})\right] & +\frac{1}{2} h_{x x}(\widetilde{K}, \widetilde{K})\left[E \Delta_{1}^{2}(\widetilde{K})+E \Delta_{2}^{2}(\widetilde{K})\right] \\
& +h_{x y}(\widetilde{K}, \widetilde{K}) E\left[\Delta_{1}(\widetilde{K}), \Delta_{2}(\widetilde{K})\right] \\
= & \\
= &
\end{aligned}
$$

Menurut Goedhart dkk (2016) untuk mendapatkan $c$ yang diinginkan maka, dengan menggunakan metode perluasan dua langkah dari Taylor dan,

1. Mengganti $\widetilde{K}=K+c$

2. Menghilangkan nilai $c^{2}, c E \Delta_{1}(K)$ dan $c E \Delta_{2}(K)$ karena dianggap kecil.

3. Aproksimasi $h(\widetilde{K}, \widetilde{K})$ dengan $h(K, K)+c\left[h_{x}(K, K)+h_{y}(K, K)\right]$ dan $h_{x}(K, K)=$ $h_{y}(K, K)$ maka, $\operatorname{Eh}\left(\widetilde{K}+\Delta_{1}(\widetilde{K}), \widetilde{K}+\Delta_{2}(\widetilde{K})\right)$ 


$$
\begin{aligned}
\approx h(K, & K)+c\left[h_{x}(K, K)+h_{y}(K, K)\right]+h_{x}(K, K)\left[E \Delta_{1}(K)+E \Delta_{2}(K)\right] \\
& +\frac{1}{2} h_{x x}(K, K)\left[E \Delta_{1}^{2}(K)+E \Delta_{2}^{2}(K)\right]+h_{x y}(K, K) E\left[\Delta_{1}(K), \Delta_{2}(K)\right] \\
& =h(K, K)
\end{aligned}
$$

Jika $\hat{\mu}$ dan $\hat{\sigma}$ pada (2.6) adalah parameter tak bias dari $\mu$ dan $\sigma$ maka, $E \Delta_{1}(K)=$ $E \Delta_{2}(K)=0$ dan $E \Delta_{1}^{2}(K)=E \Delta_{2}^{2}(K)$. Oleh karena itu nilai $c$ yang persamaan (2.5) adalah,

$$
c=-\frac{h_{x x}(K, K) E \Delta_{1}^{2}(K)+h_{x y}(K, K) E\left[\Delta_{1}(K) \Delta_{2}(K)\right]}{2 h_{x}(K, K)}
$$

Fungsi dari $h(x, y), h_{x}(x, y), h_{x x}(x, y)$ dan $h_{x y}(x, y)$ bergantung dari nilai dari fungsi $g(\alpha)$. Untuk ARL 0 , maka $g(\alpha)=\frac{1}{\alpha}$. Turunan untuk fungsi $h(x, y)$ tersebut pada titik $(K, K)$ diberikan pada Tabel 2.1.

Tabel 1. Turunan Fungsi $H$ Untuk Point $(x, y)$ Atau Khususnya Pada Point $(K, K)$ Dimana

$$
g(\alpha)=\frac{1}{\alpha}
$$

\begin{tabular}{|c|c|c|c|c|}
\hline Point & $h$ & $h_{x}$ & $h_{x x}$ & $h_{x y}$ \\
\hline \multirow{2}{*}{$(x, y)$} & $\frac{1}{\bar{\Phi}(x)+\bar{\Phi}(y}$ & $\frac{\phi(x)}{(\bar{\Phi}(x)+\bar{\Phi}(y))^{2}}$ & $\frac{2 \phi^{2}(x)}{(\bar{\Phi}(x)+\bar{\Phi}(y))^{3}}$ & $\frac{2 \phi(x) \phi(y)}{(\bar{\Phi}(x)+\bar{\Phi}(y))}$ \\
\hline$(K, K)$ & $\frac{1}{2 \bar{\Phi}(K)}$ & $\frac{\phi(K)}{4 \Phi^{2}(K)}$ & $\frac{\phi^{2}(K)}{4 \Phi^{3}(K)}-\frac{K \phi(K)}{4 \Phi^{2}(K)}$ & $\frac{\phi^{2}(3)}{4 \Phi^{3}(3)}$ \\
\hline
\end{tabular}

Sumber : Goedhart dkk (2016)

Turunan pada (2.3) sampai (2.7) menurut Goedhart dkk (2016) bersifat umum. Untuk $n>1$ maka,

$$
\begin{aligned}
& E\left[\Delta_{1}(K), \Delta_{2}(K)\right] \\
& =\frac{K^{2}}{2(m(n-1)+1)} \\
& -\frac{1}{m} \\
& E \Delta_{1}^{2}(K)=E \Delta_{2}^{2}(K) \\
& =\frac{K^{2}}{2(m(n-1)+1)} \\
& +\frac{1}{m}
\end{aligned}
$$

Untuk diagram kendali Shewhart individual $(n=1)$ maka nilai,

$$
\begin{gathered}
E\left[\Delta_{1}(K), \Delta_{2}(K)\right] \\
=K^{2}\left[\frac{0.82644 m-1.082}{(m-1)^{2}}\right] \\
-\frac{1}{m} \\
E \Delta_{1}^{2}(K)=E \Delta_{2}^{2}(K) \\
\approx K^{2}\left[\frac{0.82644 m-1.082}{(m-1)^{2}}\right] \\
+\frac{1}{m}
\end{gathered}
$$




\section{Hasil Penelitian dan Pembahasan}

Data yang digunakan yaitu brix phase mentah untuk saus kemasan sachet PT. XXX ada 53 data dengan sub kelompok $n=1$. Data dibagi menjadi 2 yaitu 45 data digunakan pada fase 1 dalam mengestimasi parameter untuk menghitung faktor koreksi yang digunakan untuk menyusun batas-batas kendali dari diagram kendali Shewhart seperti BKA, BKB serta rata-rata dari proses. Sisanya sebanyak 8 data digunakan sebagai penerapan fase 2 .

Dari penjelasan data diatas, dengan $\alpha=0,0027$ dapat diperoleh nilai $K=$ $\Phi^{-1}\left(1-\frac{0.0027}{2}\right)=\Phi^{-1}(0.99865) \approx 3$ dengan nilai estimasi dari $\mu$ dan $\sigma$ untuk faktor koreksi baru untuk $\mu$ adalah $\hat{\mu}$

$$
\mu=\hat{\mu}=\overline{\bar{Y}}=\frac{1}{m} \sum_{i=1}^{m} Y_{i}=\frac{1}{45} \times(1718.6)=38.19111
$$

Dan untuk faktor koreksi baru untuk $\sigma$ adalah $\hat{\sigma}$

$$
\sigma=\hat{\sigma}=\sigma=\frac{\overline{M R}}{\frac{2}{\sqrt{\pi}}}=\frac{0,543182}{\frac{2}{\sqrt{\pi}}}=\frac{0,543182}{\frac{2}{\sqrt{3.141593}}}=0.481382
$$

Dimana, $\overline{M R}=\frac{1}{m-1} \sum_{i=1}^{m-1}\left|Y_{i+1}-Y_{i}\right|=\frac{1}{45-1}(23.9)=0,543182$.Dengan nilai faktor koreksi c dihitung melalui,

$$
\begin{aligned}
& h_{x}(K, K)=\frac{\phi(K)}{4 \Phi^{2}(K)}=\frac{\phi(3)}{4 \Phi^{2}(3)}=\frac{0,004431848}{4(1-0,998650102)^{2}}=608.02716 \\
& h_{x x}(K, K)=\frac{\phi^{2}(K)}{4 \Phi^{3}(K)}-\frac{K \phi(K)}{4 \Phi^{3}(K)}=\frac{\phi^{2}(3)}{4 \Phi^{2}(3)}-\frac{3 \phi(3)}{4 \Phi^{2}(3)} \\
& =\frac{0,004431848^{2}}{4(1-0,998650102)^{3}}-\frac{3 \cdot 0,004431848}{4(1-0,998650102)^{2}}=172.13167 \\
& h_{x y}(K, K)=\frac{\phi^{2}(K)}{4 \Phi^{3}(K)}=\frac{\phi^{2}(3)}{4 \Phi^{3}(3)}=\frac{0,004431848^{2}}{4(1-0,998650102)^{3}}=1996.21314 \\
& E \Delta_{1}^{2}(K)=K^{2}\left[\frac{0.82644 m-1.082}{(m-1)^{2}}\right]+\frac{1}{m}=3^{2}\left[\frac{0.82644 \cdot 45-1.082}{(45-1)^{2}}\right]+\frac{1}{45}=0.19007 \\
& \quad E\left[\Delta_{1}(K) \Delta_{2}(K)\right]=K^{2}\left[\frac{0.82644 m-1.082}{(m-1)^{2}}\right]-\frac{1}{m}=3^{2}\left[\frac{0.82644 \cdot 45-1.082}{(45-1)^{2}}\right]-\frac{1}{45} \\
& =0.14563
\end{aligned}
$$

Dengan,

$c=-\frac{h_{x x}(K, K) E \Delta_{1}^{2}(K)+h_{x y}(K, K) E\left[\Delta_{1}(K) \Delta_{2}(K)\right]}{2 h_{x}(K, K)}$

$$
=-\frac{172.13167 \cdot 0.19007+1996.21314 \cdot 0.14563}{2 \cdot 608.02716}
$$

Dari perhitungan $\hat{\mu}$ dan $\hat{\sigma}$ untuk faktor koreksi Goedhart kita dapat menghitung batas kontrol diagram Shewhart dimana $\widetilde{K}=K+c=3+(-0.26596)=2.7340$ dengan nilai dari batas kontronya adalah

$$
\begin{aligned}
& \widehat{U C L}=\hat{\mu}+\widetilde{K} \cdot \frac{\hat{\sigma}}{\sqrt{n}}=38.19111+2.5145 \cdot \frac{0.481382}{\sqrt{1}}=39.50723 \\
& \widehat{L C L}=\hat{\mu}-\widetilde{K} \cdot \frac{\hat{\sigma}}{\sqrt{n}}=38.19111-2.5145 \cdot \frac{0.481382}{\sqrt{1}}=36.87499
\end{aligned}
$$




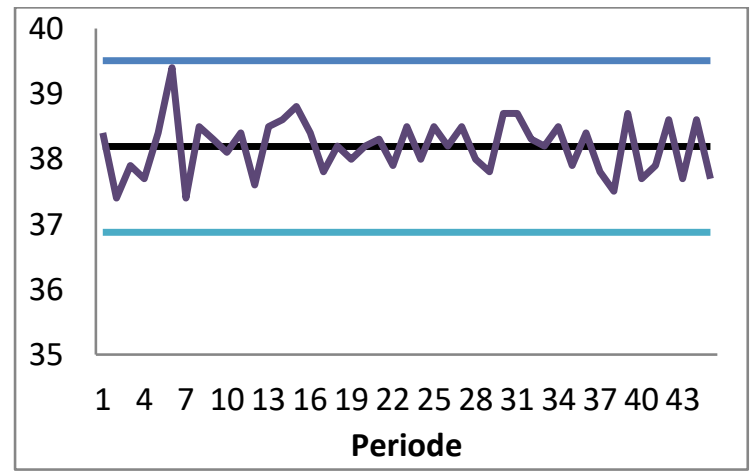

Gambar 1. Fase 1 Diagram Kendali Shewhart Data Brix Saus Sachet Phase Mentah PT. XXX

Gambar 1 merupakan diagram kendali tahap fase 1. Dari gambar 1 terlihat bahwa diagram kendali berada dalam batas kendali dan terlihat data tidak membentuk pola, maka dari itu diagram kendali dapat dipakai untuk data pada tahap fase 2.

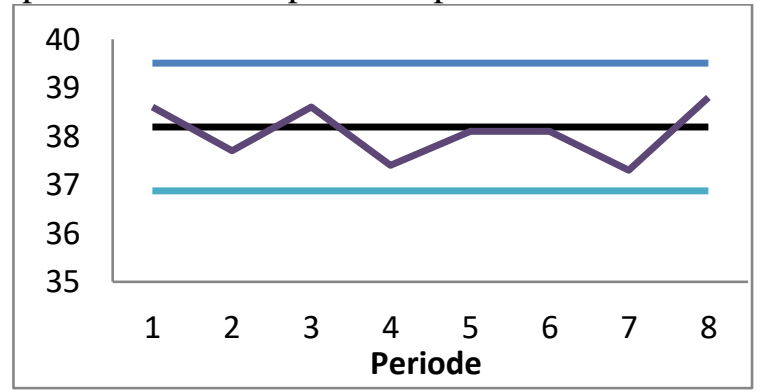

Gambar 2. Fase 2 Diagram Kendali Shewhart Data Brix Saus Sachet Phase Mentah PT. XXX

Gambar 2 merupakan diagram kendali tahap fase 2. Dari gambar 2 terlihat bahwa diagram kendali berada dalam batas kendali dan data terlihat tidak membentuk pola maka dari itu dapat disimpulkan bahwa data brix saus (kekentalan saus) sachet di PT. XXX terkendali.

\section{Kesimpulan}

Data brix saus (kekentalan saus) berdistribusi normal. Faktor koreksi diagram kendali Shewhart menggunkan metode Goedhart adalah $c=-0.26596$. Diagram kendali Shewhart berdasarkan faktor koreksi Goedhart mempunyai batas kendali atas $=39.50723$, batas kendali bawah $=$ 36.87499 serta rata-rata $=38.19111$. Dari implementasi faktor koreksi terhadap data brix (kekentalan) saus sachet phase mentah pada fase 1 dan fase 2 diperoleh bahwa proses dalam kendali.

\section{Daftar Pustaka}

[1]Albers W. dan W.C.M. Kallenberg. 2005. "New Corrections for Old Control Charts". Quality Engineering 17: 467-473

[2]Goedhart Rob, Marit S, dan Ronald J.M.M.D. (2016). Correction factor for Shewhart X and $\bar{X}$ control charts to achieve desired unconditional ARL. International journal of production research, Vol.54 No.24 7464-7479, http://dx.doi.org/10.1080/00207543.2016.1193251

[3]Meri Mufrida, Irsan, Hendi W., (2017). Analisis pengendalian kualitas pada produk SMS (Sumber Minuman Sehat) dengan metode statistical process control (SPC) studi kasus pada PT. Agrimitra Utama Persada Padang. Jurnal Teknologi Vol.7, No.1, Hal 119-126, E-ISSN : 2541-1535 ISSN:2301-4474

[4]Montgomery, C Douglas., (1990). Pengantar Pengendalian Kualitas Statistika, Gajah Mada University Press, Yogyakarta.

[5]Montgomery, C Douglas., (2009). Introduction to statistical quality control Sixth Edition, John 
Wiley\&Sons, New York.

[6]Muchlis, Dachlan R., (2010). Pengendalian Kualitas Statistika, Pustaka Ceria Yayasan Pena, Bandung.

[7]Purnomo, Rochmat A., (2016). Analisis Statistika Ekonomi dan Bisnis Dengan SPSS, Wade Group, Ponorogo.

[8]Rahmah, Afifa N, Gandhi P., (2017). Aplikasi Statistical Process Control (SPC) dalam pengendalian kualitas produksi susu di PT. Ultra Peternakan Bandung Selatan. Journal od Accounting Business Studies. Vol.2 No.1.

[9]Siegel, Sidney., (2011). Statistika Nonparametrik, PT.Gramedia, Jakarta

[10]Sudjana., (2005). Metode Statistika, Tarsito, Bandung. 